\section{6 OPEN ACCESS}

\title{
Long-term safety of pegloticase in chronic gout refractory to conventional treatment
}

\author{
Michael A Becker, ${ }^{1}$ Herbert S B Baraf, ${ }^{2}$ Robert A Yood, ${ }^{3}$ Aileen Dillon, ${ }^{4}$ \\ Janitzia Vázquez-Mellado, ${ }^{5}$ Faith D Ottery, ${ }^{6}$ Dinesh Khanna, ${ }^{7}$ John S Sundy ${ }^{8}$
}

Handling editor Tore K Kvien

- Additional supplementary files are published online only. To view these files please visit the journal online (http://dx.doi. org/10.1136/annrheumdis2012-201795).

${ }^{1}$ Rheumatology Section, The University of Chicago, Chicago, Illinois, USA

${ }^{2}$ Center for Rheumatology and Bone Research, Wheaton, Maryland, USA

${ }^{3}$ Reliant Medical Group, Worcester, Massachusetts USA

${ }^{4}$ Rheumatology Section, Kaiser Permanente Medical Center, San Francisco, California, USA ${ }^{5}$ Department of Rheumatology, Hospital General de Mexico, Mexico City, Mexico

${ }^{6}$ Savient Pharmaceuticals, Inc., East Brunswick, New Jersey, USA

${ }^{7}$ Department of Medicine, University of Michigan, Ann Arbor, Michigan, USA

${ }^{8}$ Duke Clinical Research Unit, Duke University Medical Center, Durham, North

Carolina, USA

\section{Correspondence to}

Dr Michael A Becker, 237 East Delaware Pl, Chicago, IL 60611-1713, USA; mbecker@ medicine.bsd.uchicago.edu

Accepted 9 August 2012 Published Online First 10 November 2012

\section{ABSTRACT}

Objective To evaluate the long-term safety (up to 3 years) of treatment with pegloticase in patients with refractory chronic gout.

Methods This open-label extension (OLE) study was conducted at 46 sites in the USA, Canada and Mexico. Patients completing either of two replicate randomised placebo-controlled 6-month trials received pegloticase $8 \mathrm{mg}$ every 2 weeks (biweekly) or every 4 weeks (monthly). Safety was evaluated as the primary outcome, with special interest in gout flares and infusion-related reactions (IRs). Secondary outcomes included uratelowering and clinical efficacy.

Results Patients $(n=149)$ received a mean \pm SD of 28 \pm 18 pegloticase infusions and were followed for a mean of $25 \pm 11$ months. Gout flares and IRs were the most frequently reported adverse events; these were least common in patients with a sustained urate-lowering response to treatment and those receiving biweekly treatment. In 10 of the 11 patients with a serious IR, the event occurred when uric acid exceeded $6 \mathrm{mg} / \mathrm{dl}$. Plasma and serum uric acid levels remained $<6 \mathrm{mg} / \mathrm{dl}$ in most randomised controlled trial (RCT)-defined pegloticase responders throughout the OLE study and were accompanied by sustained and progressive improvements in tophus resolution and flare incidence.

Conclusions The safety profile of long-term pegloticase treatment was consistent with that observed during 6 months of RCT treatment; no new safety signals were identified. Improvements in clinical status, in the form of flare and tophus reduction initiated during RCT pegloticase treatment in patients maintaining goal range urate-lowering responses were sustained or advanced during up to 2.5 years of additional treatment.

\section{INTRODUCTION}

Urate-lowering therapy is the mainstay of chronic gout management and aims at achieving and maintaining sub-saturating serum uric acid (SUA) concentrations, most often recommended as $\leq 0.36 \mathrm{mmol} / 1$ $(\leq 6 \mathrm{mg} / \mathrm{dl}) .{ }^{1}{ }^{2}$ Long-term maintenance of goal range SUA is associated with depletion of urate crystals in synovial fluid $^{3}{ }^{4}$ and reductions in tophus size and flare frequency. ${ }^{5-7}$ Because the rates of achieving these improvements are related to the degree of urate-lowering, ${ }^{4} 68$ the optimal therapeutic target may be substantially lower than $6 \mathrm{mg} / \mathrm{dl}$ in patients with severe manifestations of chronic gout, ${ }^{5}$ prompting one recommendation for a target SUA of $<5 \mathrm{mg} / \mathrm{dl}^{9}{ }^{9}$
Up to $3 \%$ of the estimated eight million patients with gout in the USA ${ }^{10}$ fail urate-lowering management with standard-of-care therapies (oral xanthine oxidase inhibitors) because of drug intolerance/ contraindication or treatment refractoriness. ${ }^{11-13}$ Such patients are at risk of progression to increasing flare recurrences, gouty arthropathy, destructive and deforming tophi and chronic pain, frequently accompanied by impaired physical function and poor health-related quality of life. Until recently these patients had few or no effective uratelowering options to prevent or reverse gout progression. Pegloticase, a mammalian recombinant uricase conjugated to monomethoxypoly(ethylene glycol) (mPEG), was developed as an enzymatic alternative for the treatment of patients with gout refractory to conventional oral therapies and was approved in the USA in 2010. ${ }^{14-16}$ When administered intravenously, pegloticase reduces the urate concentration in the intravascular space to below the limit of solubility $(6.8 \mathrm{mg} / \mathrm{dl})$. The resulting reduction in extracellular soluble urate concentration is hypothesised to favour dissolution of deposited urate crystals, resulting in progressive normalisation of body urate pools and improvements in clinical signs and symptoms of gout. ${ }^{17} 18$

The tolerability and efficacy of pegloticase treatment in patients with refractory chronic gout were demonstrated in replicate 6-month randomised double-blind placebo-controlled trials (RCTs). ${ }^{17}$ Pegloticase administered every 2 weeks (biweekly) or every 4 weeks (monthly) produced treatment responses (plasma uric acid (PUA) $<6 \mathrm{mg} / \mathrm{dl}$ for $\geq 80 \%$ of the time during months 3 and 6 ) in $42 \%$ and $35 \%$ of patients, respectively, compared with $0 \%$ for patients receiving placebo. PUA normalised within $24 \mathrm{~h}$ of the first pegloticase infusion in all patients, but in non-responders the urate-lowering response was lost over time. Infusion-related reactions (IRs) were the most common reason for discontinuations in the RCTs $(10 \%$ of biweekly treated patients and $13 \%$ of monthly treated patients). ${ }^{17}$ This report focuses on the long-term open-label extension (OLE) of the RCTs which provided an additional 2.5 years of safety data.

\section{METHODS}

\section{Study design and patients}

The OLE (NCT01356498) enrolled patients at 46 centres in the USA, Canada and Mexico who had completed either of the two RCTs (NCT01356498, NCT00325195). ${ }^{17}$ The OLE was conducted from 
December 2006 to July 2009; compliance and permission information is provided in the online supplementary material. Protocol amendments extended the OLE from 12 months to a maximum of 30 months. Figure 1 summarises the OLE study design, inclusion criteria (exclusion criteria are presented in the online supplementary material), end points and evaluations. Delays in treatment between the randomised trials and the OLE resulted from administrative requirements, such as time for Institutional Review Board approvals and site implementation. Except where specifically indicated, patients were categorised for analysis purposes according to the first dosing regimen administered during the OLE study.

Details of safety evaluations and prophylaxis regimens for IRs and flares are provided in the online supplementary material. As IRs were one of the events of interest, physical examinations and monitoring of vital signs were performed at the time of all IRs and medical intervention was provided as appropriate. For IRs occurring during infusions, the infusion could be discontinued, slowed by half, or interrupted and later restarted at a slower rate.

Immunogenicity was assessed from serum collected every 12 weeks using a validated ELISA to detect IgG and IgM pegloticase antibody. Antibody titres were categorised as low $(\leq 1$ : 2430 ) or high ( $>1: 2430)$, consistent with the RCTs. ${ }^{17}$ Tophus complete response was defined as $100 \%$ reduction in the measured area of at least one tophus of baseline diameter $\geq 5 \mathrm{~mm}$ without growth of any other baseline tophus or appearance of any new tophus.

\section{Statistical analysis}

Safety and efficacy end points were evaluated using descriptive statistics in the intent-to-treat population, which included all patients who received at least one dose of pegloticase during the OLE study and had follow-up data. Demographic and baseline clinical values are described in the online supplementary material.

\section{RESULTS}

\section{Patient disposition}

A total of 225 patients were enrolled in the two RCTs and 212 patients were included in the primary efficacy analysis. Of these, 157 patients (74\%) completed the RCTs and 151 (96\% of completers) entered the OLE study, including 57 (97\%) patients from the biweekly pegloticase group, 55 (93\%) from the monthly pegloticase group and all 39 patients from the placebo group (figure 2). All patients received pegloticase in the OLE study, except for two patients who chose observation only after receiving monthly pegloticase in the RCTs. For patients treated with pegloticase in the RCTs, a higher proportion of those defined as responders than those defined as nonresponders completed the OLE study, as did a higher proportion of patients allocated to placebo in the RCTs who then received biweekly pegloticase compared with those who received monthly pegloticase (figure 2). The most common reasons for discontinuing treatment during the OLE study were adverse events (AEs) in 18\% (27/149) of patients and loss of uratelowering response in $9 \%(13 / 149)$.

\section{Patients}

The OLE study population had a mean age of 56.8 years (range $30-89)$ and $79 \%$ were men. Most patients were white (69\%), African-American (13\%) or Hispanic/Latino (11\%). Risk factors were common; the most common were cardiovascular $(94 \%$ of patients had at least one cardiovascular risk factor, most commonly hypertension, $72 \%$ ), obesity (body mass index (BMI) $\geq 30 \mathrm{~kg} / \mathrm{m}^{2}, 65 \%$ ) and dyslipidaemia (52\%). Chronic kidney disease (creatinine clearance $<1.0 \mathrm{ml} / \mathrm{s}$ ) was present in $26 \%$ of patients. Less commonly occurring risk factors and concomitant medications are described in the online supplementary material.

\section{Exposure to pegloticase}

Patients received a mean of 28 pegloticase infusions (median 26; range 1-59) during the OLE study, with RCT responders receiving more infusions than non-responders (mean 35 vs 26). When exposure to pegloticase was pooled from the RCT and OLE studies, patients received a mean of 35 infusions (range 1-70). Thirty of 67 patients (45\%) who started on monthly pegloticase switched to biweekly treatment at some point during the OLE study; only $12 \%$ (10/82) switched from biweekly to monthly treatment. Overall, patients remained in the OLE study for a mean of 25 months (median 29 months; range 0-37 months, including a mandated end-of-study observation period (no treatment) for a maximum of 6 months).

\section{Safety}

\section{Adverse events (AEs)}

Nearly all patients (98\%) had at least one AE during the OLE study (table 1). The overall incidence of AEs did not differ between responders and non-responders from the RCTs or for patients initially on placebo who started pegloticase biweekly

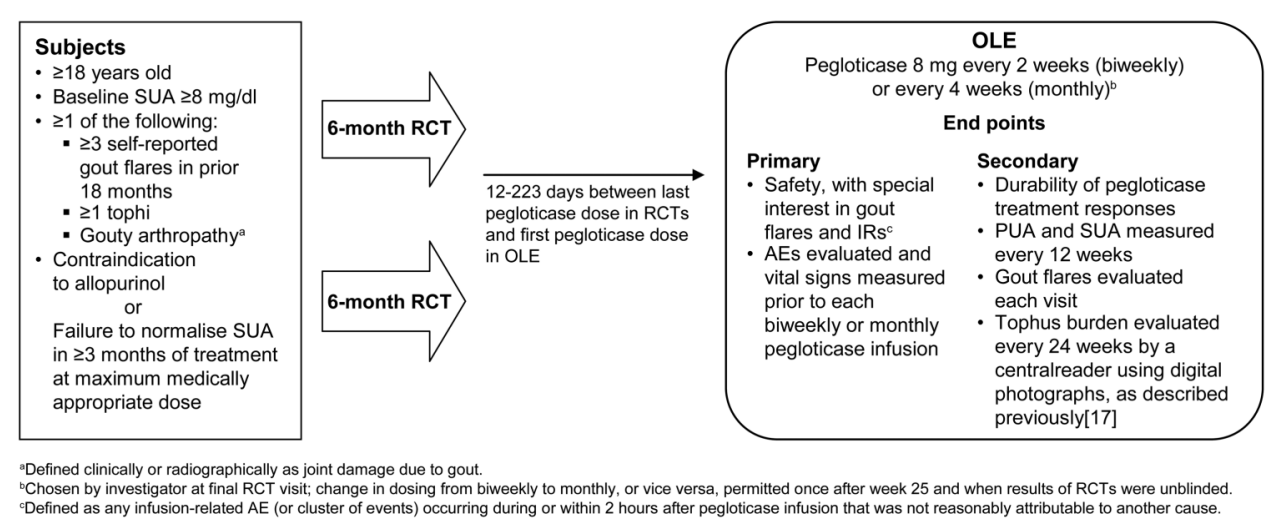

Figure 1 Study design schematic for the open-label extension study. AE, adverse event; IR, infusion-related reaction; OLE, open-label extension; PUA, plasma uric acid; RCT, randomised controlled trial; SUA, serum uric acid. 


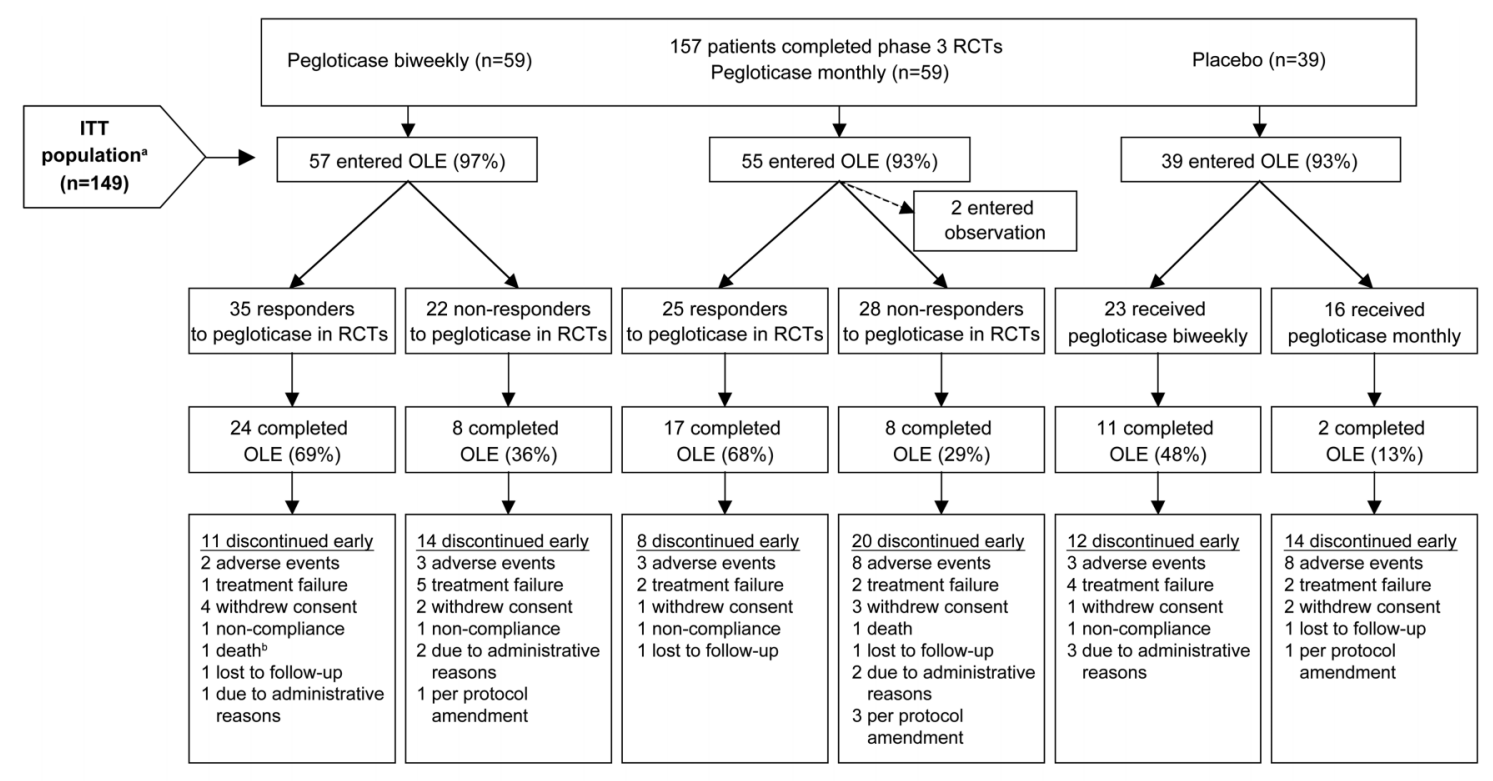

aThe intent-to-treat population consisted of all patients who entered the OLE, received at least one dose of pegloticase, and had follow-up data. bPatient died $>30$ days after last dose of pegloticase, but did not formally enroll in the observation period.

Figure 2 Disposition for all patients entering the open-label extension (OLE) study. ITT, intention-to-treat; RCT, randomised controlled trial.

versus monthly. Both gout flares and IRs occurred at a lower rate with the biweekly regimen during the OLE study $(2.7$ per patient per year vs 4.7 with the monthly regimen and 1.3 per patient per year vs 2.1 , respectively).

The incidence of gout flares during the OLE study was lowest for responders who had received biweekly pegloticase in the RCTs and highest for RCT non-responders to monthly pegloticase and patients treated with placebo in the RCTs. The incidence of IRs in the OLE study was lowest for RCT responders regardless of pegloticase schedule and highest for RCT non-responders to monthly pegloticase and RCT placebo patients starting monthly pegloticase. Eleven patients (7\%) discontinued pegloticase due to AEs, including seven patients with IRs; six of these seven patients were RCT pegloticase non-responders or RCT placebo-treated patients. Among all patients with AEs judged as treatment-related by the investigators $(66 \%, 99 / 149)$, only gout flares and IRs were reported in $>5 \%$ of patients.

Most AEs (53\% of patients) were investigator-rated as moderate in intensity. Overall, 36\% (54/149) of patients had severe AEs, of which $17 \%$ were treatment-related, most commonly IRs $(7 \%)$ or gout flares $(7 \%)$. Of note, no RCT pegloticase responder had a severe treatment-related IR or a severe gout flare. The highest rates of severe IRs and flares (26\% for both; $6 / 23$ ) were reported in patients treated with biweekly pegloticase during the OLE study who had received placebo in the RCTs. Assessments of haematology, clinical chemistry and urinalysis identified no significant change from baseline (except for UA) in any of the subgroups defined by response to pegloticase or pegloticase administration schedule.

Twenty-four adjudicated cardiovascular events in 21 patients were identified in the OLE study by the independent expert committee. These events occurred in responders and nonresponders with no apparent relationship to the duration of treatment or time since the last pegloticase infusion. Eight of these events occurred in patients who were being observed and had not received pegloticase for $>30$ days.
Serious AEs and deaths

Approximately one-third of patients (34\%, 51/149) experienced a total of 106 serious AEs during the OLE study. Thirteen patients who reported serious AEs discontinued treatment. Among the 13 serious AEs considered possibly related to study drug, there were 11 IRs (one serious AE of IR was judged unlikely to be related to the study drug), one skin necrosis (severe) and one nephrolithiasis (moderate). Among the 11 serious AEs of IR, all but one occurred when the UA values

Table 1 Summary of AEs during the OLE study

\begin{tabular}{lc}
\hline AEs in the OLE study & $\begin{array}{c}\text { All treated patients } \\
\text { (N=149) } \\
\mathbf{N}(\%)\end{array}$ \\
\hline Subjects with any AE & $146(98)$ \\
Subjects with any serious AE & $51(34)$ \\
Subjects with serious AEs considered related to & $13(9)$ \\
pegloticase & \\
Discontinuations due to AE & $11(7)$ \\
Most common AEs (incidence >10\%) & \\
Gout flare & $106(71)$ \\
Infusion-related reaction & $65(44)$ \\
Arthralgia & $29(20)$ \\
Upper respiratory tract infection & $27(18)$ \\
Pain in extremity & $26(17)$ \\
Back pain & $25(17)$ \\
Diarrhoea & $22(15)$ \\
Peripheral oedema & $21(14)$ \\
Urinary tract infection & $20(13)$ \\
Nausea & $17(11)$ \\
Headache & $16(11)$ \\
Fatigue & $15(10)$ \\
Sinusitis & $15(10)$ \\
Nasopharyngitis & $15(10)$ \\
\hline AE, adver eve &
\end{tabular}

$\mathrm{AE}$, adverse event; OLE, open-label extension. 
exceeded $6 \mathrm{mg} / \mathrm{dl}$. Four deaths occurred during the OLE study, all of which were judged as unlikely to be related to the study drug by the investigator (see online supplementary material).

IRs

During the RCTs, IRs were the second most common AE and were reported in $26 \%$ of patients receiving biweekly pegloticase and $42 \%$ of patients receiving monthly pegloticase. In the OLE, IRs were reported in $44 \%(60 / 149)$ of patients. The rate of IRs was lower in RCT responders $(17 \%, 10 / 60)$ than in nonresponders $(52 \%, 26 / 50)$ and highest among patients who received placebo in the RCT $(62 \%, 24 / 39)$. IRs were rated mild in $27 \%(16 / 60)$ of patients, moderate in $55 \%(33 / 60)$ and severe in $18 \%(11 / 60)$. IRs rated as severe in the OLE occurred in four patients, none of whom sustained goal range urate lowering, and in seven patients who received placebo in the RCT. IRs were the reason cited for withdrawal from the study for $6 \%$ (9/ 149) of patients.

Except for three patients with IRs manifesting in the $2 \mathrm{~h}$ period after infusion, all IRs occurred during infusion. The most common signs and symptoms associated with IRs were musculoskeletal pain/discomfort, flushing, erythema, nausea/ vomiting, dyspnoea, sweating, headache, blood pressure changes, urticaria and pruritus. All IRs resolved with supportive measures and no patient required intubation, mechanical ventilatory support, pressors or hospitalisation. Three patients were found to have anaphylaxis based on a retrospective analysis. Symptoms included red, itching or swollen eyes, throat irritation, musculoskeletal symptoms, skin flushing, hypotension, dizziness and vomiting.

\section{Immunogenicity}

A total of $31 \%(52 / 169)$ of patients treated with pegloticase had high pegloticase antibody titres ( $>1: 2430)$ during the RCTs. High-titre antibodies were identified in 53\% (67/127) of patients at the week 13 assessment of the OLE; this number increased by the end of the study (antibody titres $>1: 2430$ in $60 \%$; 90/149). Only one patient had evidence of in vitro neutralising antibodies against uricase activity; this was measurable at only one time point. Consistent with findings from the RCTs, ${ }^{17}$ low titres $(\leq 1: 2430)$ of anti-pegloticase antibodies were less likely to be associated with loss of SUA response (see online supplementary material for supporting data).

\section{Efficacy}

Serum uric acid response

The concordance of PUA and SUA (collected for all patients at the same time points) for values above and below the target of $6 \mathrm{mg} / \mathrm{dl}$ was $95 \%$ in samples collected during the OLE study. The efficacy data reported here focus on SUA because of its ready accessibility in clinical settings. The overall mean \pm SD SUA at baseline was $10.1 \pm 1.4 \mathrm{mg} / \mathrm{dl}$. Most responders to biweekly and monthly pegloticase in the RCTs maintained SUA $<6 \mathrm{mg} / \mathrm{dl}$ throughout the OLE study (figure $3 \mathrm{~A}$ ). Among

A

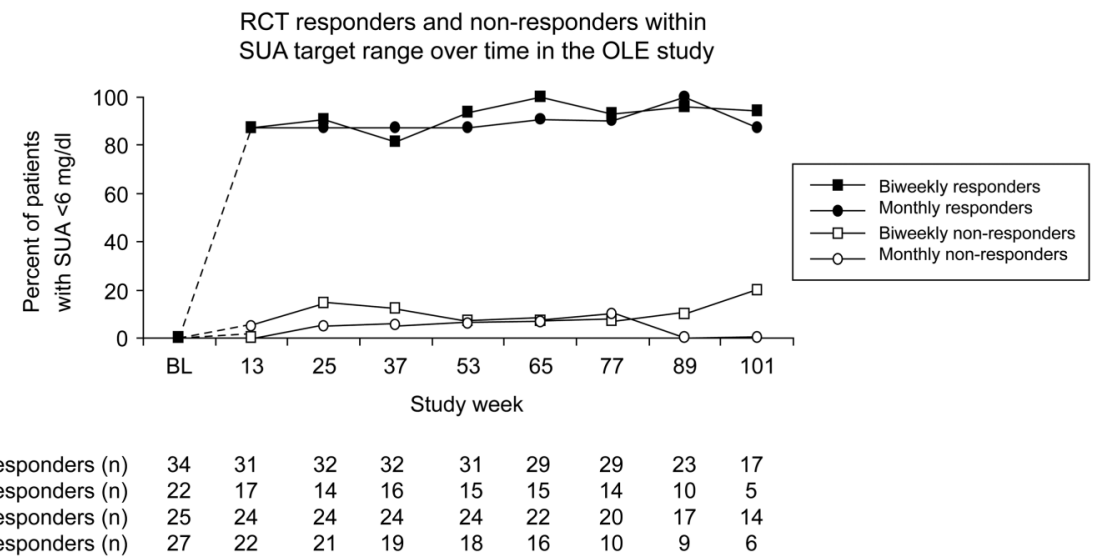

B
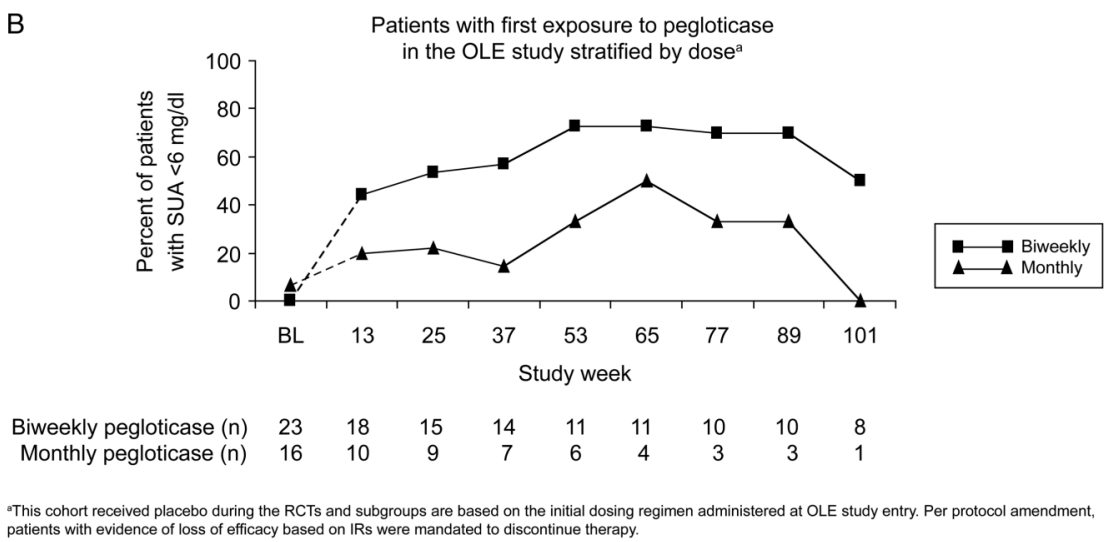

Figure 3 Percentage of patients with serum uric acid (SUA) $<6 \mathrm{mg} / \mathrm{dl}$ by study week, uric acid responder status in the randomised controlled trial (RCT) and pegloticase dose regimen. (A) All patients who entered the open-label extension (OLE) study after receiving pegloticase during the RCTs. Subgroups were defined by SUA response during the RCTs. (B) All patients who entered the OLE study after receiving placebo during the RCT and therefore had their first exposure to pegloticase in the OLE study. Subgroups are defined by the pegloticase dose administered at OLE entry. 
patients randomised to placebo in the RCTs, biweekly pegloticase administration in the OLE study produced greater reductions in mean SUA and a greater proportion of patients maintaining SUA $<6 \mathrm{mg} / \mathrm{dl}$ than monthly pegloticase (figure 3B).

\section{Tophus response}

Tophus burden continued to decrease during ongoing pegloticase therapy. At the end of the RCTs, a complete response in at least one tophus was reported for $40 \%(21 / 52)$ of patients with tophi treated with biweekly pegloticase, 21\% (11/52) of those treated with monthly pegloticase and $7 \%(2 / 27)$ of those treated with placebo. By OLE week 13 a complete response in at least one tophus was reported for $45 \%(36 / 80)$ of patients with tophi at RCT inception. At the final OLE visit (week 125), $60 \%$ (56/94) of patients with evaluable tophi had a complete response. Patients with a sustained urate-lowering response to treatment were more likely to experience complete tophus resolution. A complete response was seen in $87 \%$ (32/ 37) of patients in the responder group and in $31 \%(11 / 36)$ designated non-responders on the basis of SUA measurements in the RCTs.

As prespecified in the protocol, each patient could have up to five measurable (and two additional) target or index tophi. Responses based on the total number of tophi were assessed to complement information on the proportion of patients with tophus complete response. After 1 year of pegloticase treatment in the OLE study, 61\% (185/302) of all target tophi had completely resolved. Among patients who qualified as responders in the RCTs, a complete response of 70\% (102/145) of all target tophi was achieved after 1 year of open-label treatment.

\section{Gout flares}

Gout flares occurred in $71 \%$ of patients $(106 / 149)$ during the OLE study; the mean number of flares per patient per 3-month period was 0.5 over the duration of the trial. The highest flare rates occurred in $52 \%$ of patients (78/149; 1.1 flares/patient) during the first 3 -month period. Flare rates diminished with continued treatment in the OLE among patients who were urate-lowering responders during the RCT compared with patients who were RCT non-responders (figure 4A,B). For example, in the cohort of patients sustaining goal uratelowering on the biweekly pegloticase OLE dose schedule, flares diminished substantially (occurring in 26\% (9/35) during months $1-3$ of the OLE study, 9\% (3/32) during months 10-12 and $3 \%(1 / 29)$ during months $22-24)$.

\section{DISCUSSION}

Pegloticase is a PEGylated mammalian recombinant uricase that was developed to control hyperuricaemia and its clinical manifestations in patients with refractory chronic gout and no other urate-lowering options. In the present OLE of two RCTs, ${ }^{17}$ long-term treatment with pegloticase was safe and generally well tolerated, especially in patients who had experienced sustained goal range urate-lowering responses during blinded treatment. In addition to maintaining markedly subsaturating urate levels, these patients showed progressive clinical benefit in gout flares and tophus reduction during pegloticase treatment for an average of 2 years in the OLE study.
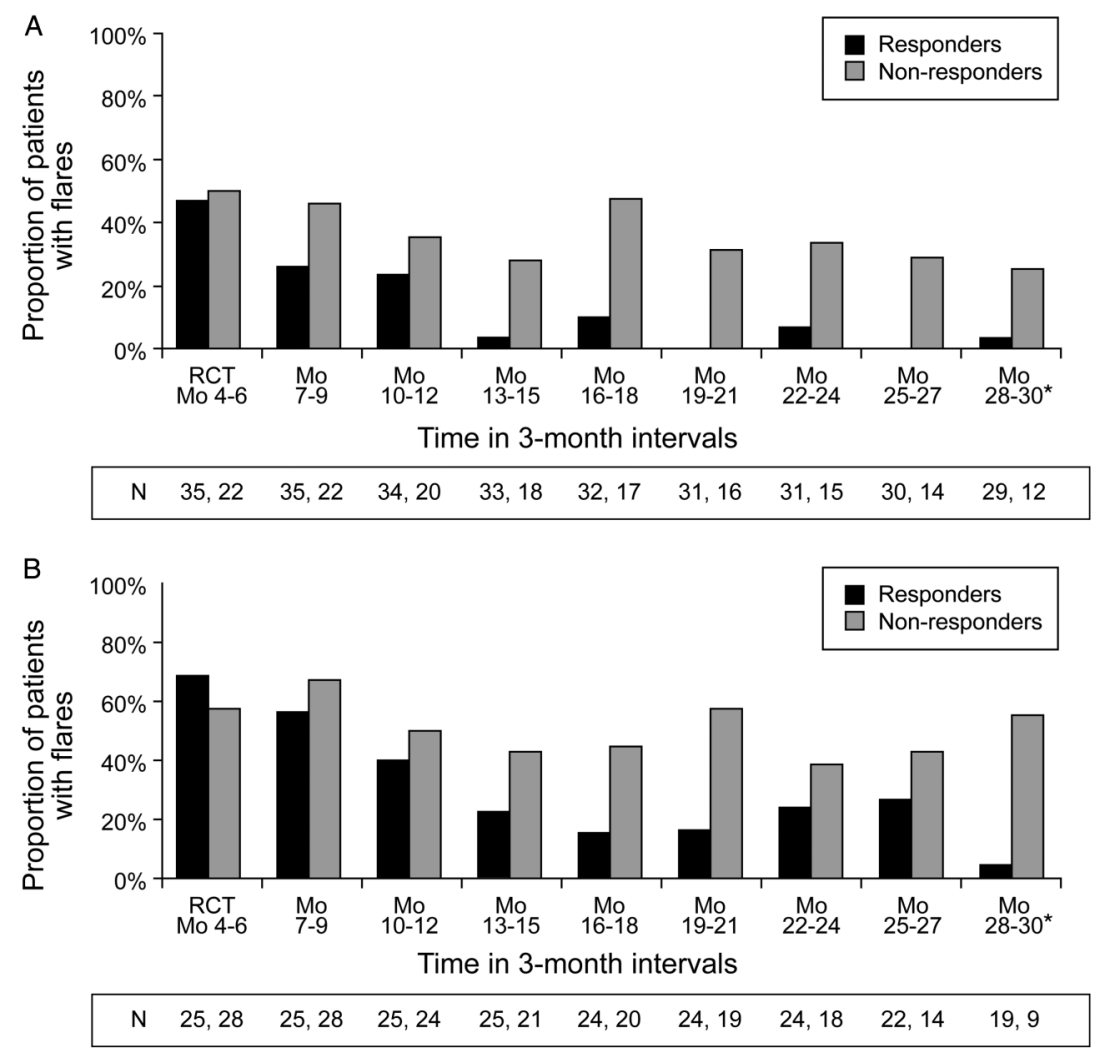

*Some of the defined subgroups contained no patients after month 30 and therefore flare data is not shown after month 30

Figure 4 Proportions of patients who received (A) biweekly pegloticase or $(\mathrm{B})$ monthly pegloticase throughout the randomised controlled trial (RCT) and open-label extension periods and reported gout flares (per 3-month interval). 
Target range SUA was achieved for $55 \%$ of all patients at week 25 of the OLE study. This is consistent with findings from the RCTs in which $42 \%$ of patients treated with biweekly pegloticase showed sustained urate lowering for 6 months. ${ }^{17}$ Conversely, patients losing urate-lowering efficacy did so within the first few months of treatment in the RCTs, and urate levels for this cohort remained above target for the duration of the OLE study. About one-third of patients who did not sustain $\mathrm{SUA}<6 \mathrm{mg} / \mathrm{dl}$ in the RCTs retained clinical benefit during the OLE study with at least one tophus remaining resolved. However, this finding could be explained by an initial response followed by a protracted period needed for sufficient reaccumulation of crystal deposits to become clinically detectable.

A post hoc analysis of the RCTs revealed relationships between loss of urate-lowering efficacy, risk of IRs and development of high-titre pegloticase antibodies. ${ }^{17}$ Because of blinding of urate levels during the RCTs, the relationship between loss of urate-lowering efficacy and the risk of pegloticase infusion reactions was not appreciated until several months after initiation of the OLE study. As a result, some patients who were RCT pegloticase non-responders continued receiving pegloticase during the OLE study, providing additional information with regard to these relationships. Among patients entering the OLE study, 71 had at least one IR during the randomised trials or the OLE study; $85 \%$ of these patients (60/71) experienced their first IR when SUA was $>6 \mathrm{mg} / \mathrm{dl}$. Overall, these data support the view that the great majority of IRs can be avoided if patients discontinue treatment when, with routine preinfusion monitoring of SUA, levels in excess of $6 \mathrm{mg} / \mathrm{dl}$ indicate a sustained loss of pegloticase urate-lowering efficacy.

There are limitations to this study. First, the open-label study design carries both well-documented value and some bias as a result of being uncontrolled. ${ }^{19}$ Second, the RCT protocol called for stratification of patients based on treatment response and dosing schedule. Although this provided the opportunity to follow safety and efficacy outcomes during the OLE study for patients with specific treatment histories, it also resulted in six distinct cohorts, each containing small numbers of patients. Nevertheless, the analyses of OLE study data indicate that patients who achieve treatment success throughout the first 6 months of treatment are likely to benefit from sustained longterm urate-lowering by pegloticase with favourable clinical responses. Conversely, patients not sustaining urate-lowering treatment responses to pegloticase infusions should not be expected to have treatment benefits (extending substantially beyond the period of urate-lowering initially achieved) and incur an increased risk of IRs. We recommend discontinuation of pegloticase therapy in such patients.

A final limitation is that patients were identified by their initial allocation to biweekly or monthly treatment in the RCTs but were allowed to change dosing frequency at two time points upon entering the OLE study. A higher proportion of patients switched from monthly to biweekly treatment than vice versa, however, indicating that most patients were receiving the US FDA-approved pegloticase biweekly regimen.

In summary, the safety profile of pegloticase in the OLE study was consistent with that observed in the RCTs ${ }^{17}$ with no evidence of new safety concerns related to long-term exposure. Efficacy findings further demonstrated that clinical improvements were durable and probably progressive during long-term therapy in patients with persistent goal range uratelowering responses to pegloticase.
Acknowledgements The authors thank all the investigators and patients who participated in the pegloticase trials. Members of the Cardiovascular Event Adjudication Committee were William B White (chair), Glen E Cooke and Philip Gorelick who were compensated for their participation. The authors wish to acknowledge writing and editorial support from the $\mathrm{fm} P$ group of Fallon Medica LLC, funded by Savient Pharmaceuticals, in the preparation of this manuscript.

Contributors MAB, HSBB, RAY, AD, JV-M, FDO, DK and JSS all made substantial contributions to the analysis and interpretation of the data, contributed to the drafting of the article or revising it critically for important intellectual content and gave their final approval of the version to be published.

Funding This study was sponsored by Savient Pharmaceuticals.

Competing interests Savient Pharmaceuticals has licensed worldwide rights to the technology related to pegloticase from Duke University and Mountain View Pharmaceuticals.

Ethics approval The protocol, its amendments and informed consents were approved by a local institutional review board or by a central institutional review board (IntegReview).

Provenance and peer review Not commissioned; externally peer reviewed.

Open Access This is an Open Access article distributed in accordance with the Creative Commons Attribution Non Commercial (CC BY-NC 3.0) license, which permits others to distribute, remix, adapt, build upon this work non-commercially, and license their derivative works on different terms, provided the original work is properly cited and the use is non-commercial. See: http://creativecommons.org/licenses/by-nc/3.0/

\section{REFERENCES}

1. Zhang W, Doherty M, Bardin T, et al. EULAR evidence based recommendations for gout. Part II: management. Report of a task force of the EULAR Standing Committee for International Clinical Studies Including Therapeutics (ESCISIT). Ann Rheum Dis 2006;65:1312-24.

2. Hamburger M, Baraf HS, Adamson TC III, et al. Recommendations for the diagnosis and management of gout and hyperuricemia. Postgrad Med 2011;123(6 Suppl 1):3-36.

3. Li-Yu J, Clayburne G, Sieck M, et al. Treatment of chronic gout. Can we determine when urate stores are depleted enough to prevent attacks of gout? J Rheumatol 2001;28:577-80.

4. Pascual E, Sivera F. Time required for disappearance of urate crystals from synovial fluid after successful hypouricaemic treatment relates to the duration of gout. Ann Rheum Dis 2007;66:1056-8.

5. Perez-Ruiz F, Lioté F. Lowering serum uric acid levels: what is the optimal target for improving clinical outcomes in gout? Arthritis Rheum 2007:57:1324-8.

6. Shoji A, Yamanaka H, Kamatani N. A retrospective study of the relationship between serum urate level and recurrent attacks of gouty arthritis: evidence for reduction of recurrent gouty arthritis with antihyperuricemic therapy. Arthritis Rheum 2004:51:321-5.

7. Becker MA, Schumacher HR, MacDonald PA, et al. Clinical efficacy and safety of successful longterm urate lowering with febuxostat or allopurinol in subjects with gout. J Rheumatol 2009;36:1273-82.

8. Perez-Ruiz F, Calabozo M, Pijoan Jl, et al. Effect of urate-lowering therapy on the velocity of size reduction of tophi in chronic gout. Arthritis Rheum 2002;47:356-60.

9. Jordan KM, Cameron JS, Snaith M, et al. British Society for Rheumatology and British Health Professionals in Rheumatology guidelines for the management of gout. Rheumatology (Oxford) 2007:46:1372-4.

10. Zhu Y, Pandya BJ, Choi HK. Prevalence of gout and hyperuricemia in the US general population: the National Health and Nutrition Examination Survey 2007-2008. Arthritis Rheum 2011;63:3136-41.

11. Edwards NL. Treatment-failure gout: a moving target (editorial). Arthritis Rheum 2008:58:2587-90.

12. Brook RA, Forsythe A, Smeeding JE, et al. Chronic gout: epidemiology, disease progression, treatment and disease burden. Curr Med Res Opin 2010;26:2813-21.

13. Doghramji PP, Edwards NL, McTigue J. Managing gout in the primary care setting what you and your patients need to know. Am J Med 2010;123:S2

14. Sherman MR, Saifer MG, Perez-Ruiz F. PEG-uricase in the management of treatment-resistant gout and hyperuricemia. Adv Drug Deliv Rev 2008;60:59-68.

15. Krystexxa ${ }^{\circledR}$ (pegloticase) Injection, for intravenous infusion. Prescribing information. East Brunswick, NJ: Savient Pharmaceuticals, 2010.

16. Schlesinger N, Yasothan U, Kirkpatrick P. Pegloticase. Nat Rev Drug Discov 2011:10:17-18.

17. Sundy JS, Baraf HS, Yood RA, et al. Efficacy and tolerability of pegloticase for the treatment of chronic gout in patients refractory to conventional treatment. JAMA 2011;306:711-20.

18. Richette P, Bardin T. Gout. Lancet 2010;375:318-28.

19. Day RO, Williams KM. Open-label extension studies: do they provide meaningful information on the safety of new drugs? Drug Safety 2007;30:93-105. 